\title{
Does angina vary with the menstrual cycle in women with premenopausal coronary artery disease?
}

\author{
G W Lloyd, N R Patel, E McGing, A F Cooper, D Brennand-Roper, G Jackson
}

\begin{abstract}
Objective-To determine whether angina in women with established coronary heart disease varies with changes in hormone concentrations during the menstrual cycle.

Design-Subjects were prospectively studied once a week for four weeks.

Setting-Cardiology outpatient department of tertiary referral centre.

Subjects-Nine premenopausal women, mean (SEM) age 38.89 (2.18) years, with established coronary heart disease, symptomatic angina, and a positive exercise test.

Main outcome measure-Myocardial ischaemia as determined by time to $1 \mathrm{~mm}$ ST depression during symptom limited exercise testing. Position in the menstrual cycle was established from hormone concentrations.

Results-The early follicular phase, when oestradiol and progesterone concentrations were both low, was associated with the worst exercise performance in terms of time to onset of myocardial ischaemia, at 290 (79) seconds; the best performance (418 (71) seconds) was when oestrogen concentrations were highest in the mid-cycle $(\mathrm{p}<0.05)$. Similar trends were observed in other measured variables. Progesterone concentrations did not influence exercise performance.

Conclusions-During the menstrual cycle myocardial ischaemia was more easily induced when oestrogen concentrations were low. This may be important for timing the assessment and evaluating treatment in women with coronary heart disease.

(Heart 2000;84:189-192)
\end{abstract}

Keywords: myocardial ischaemia; exercise testing; oestradiol; progesterone

The vascular effects of $17-\beta$ oestradiol may be important in the presence of myocardial ischaemia. Oestradiol appears to act as a vasodilator. Various mechanisms have been proposed for this, including restoration of impaired endothelial function, ${ }^{1}$ calcium antagonism, ${ }^{2}$ alterations in sympathetic tone, ${ }^{3}$ and angiotensin converting enzyme inhibition. ${ }^{4}$ Several investigators have attempted to show an antianginal effect of postmenopausal oestrogen replacement treatment, but with mixed results. ${ }^{5-7}$

Coronary heart disease is rare before the menopause, partly owing to the protective effect of female sex hormones. There are, however, important physiological changes related to cyclical fluctuations in oestradiol that effect vascular tone. ${ }^{89}$ Furthermore, during the different phases of the menstrual cycle variance in the severity of several conditions-including migraine, ${ }^{10}$ asthma, ${ }^{11}$ and cardiac arrhythmias $^{12}$ - is well documented. In one case report, pronounced manifestations of ischaemic heart disease associated with vasospasm were noted, with variation in the menstrual cycle, and it has been suggested that angina in the absence of obstructive coronary disease (syndrome $\mathrm{X}$ ) is also affected by the menstrual cycle. ${ }^{13}$ In order to clarify the role of the different female hormones in patients with angina and angiographically documented coronary artery disease, we investigated the effect of menstrual periodicity on the severity of angina and exercise performance using the exercise treadmill ECG.

\section{Methods}

We examined women who were premenopausal, with regular monthly menses and a follicular stimulating hormone (FSH) concentration $<13 \mathrm{U} / 1$ and luteinising hormone (LH) $<43 \mathrm{U} / 1$ on all four visits. Inclusion criteria were the presence of angiographically significant coronary heart disease $(>50 \%$ diameter stenosis), symptomatic angina, and a positive exercise test. Patients taking oral contraceptives or who were pregnant were excluded, as were women with ECG abnormalities (left bundle branch block, left ventricular hypertrophy, or digoxin treatment), which would make interpretation of the treadmill ECG unreliable. Women were randomly started on the trial protocol irrespective of their position in the menstrual cycle. Before each exercise test blood was drawn for oestradiol, progesterone, $\mathrm{LH}$, and FSH. Antianginal treatment was left unchanged throughout the study.

Exercise treadmill testing was performed using a Marquette MAX I apparatus (Marquette Medical Systems, Milwaukee, USA) with automatic signal averaging of ST segments. We used the symptom limited Bruce protocol. Blood pressure was measured at one minute intervals. ST segment depression was considered to be significant if there was $1 \mathrm{~mm}$ of planar or downsloping ST depression in any two consecutive leads lasting for 10 or more beats. The same leads with initial ST depression were used for each subsequent evaluation. Two experienced investigators who were unaware of the menstrual or symptomatic status of the 
subject reported the exercise test. The exercise test was repeated at the same time of day in the same ambient conditions on four consecutive weeks. The study was approved by the local ethics committee, and all patients gave their written consent.

No formal power calculation was performed because of the lack of previous work in the area; however, a target population of 10 patients was envisaged from the work with postmenopausal oestrogen in women with angina. ${ }^{5}$ The population was then subdivided according to the phase in the menstrual cycle (early follicular, late follicular, early luteal, late luteal). Position in the menstrual cycle was defined as weeks from the early follicular phase (low oestrogen, low progesterone) and confirmed by appropriate hormone concentrations at each of the other visits.

Data were analysed using the SPSS for windows statistical package. Data are presented as mean (SEM). Two way comparisons were performed using paired or unpaired $t$ tests as appropriate. Group trends were assessed using analysis of variance for repeated measures. All data fulfilled parametric assumptions as defined by Levine's test. Probability values of $p \leqslant 0.05$ were considered significant.

\section{Results}

RECRUITMENT

Twelve patients were initially recruited. Two had hormone concentrations which were in the postmenopausal range at one of the four visits and were therefore excluded. One patient, although showing ST depression during a preliminary exercise test, failed to show any abnormalities on tests during the trial period and so was also excluded. This woman also had an ejection fraction of less than $25 \%$.

\section{CHARACTERISTICS}

The other nine patients were aged 38.89 (2.18) years. Four of the women had suffered a previous myocardial infarct and one had undergone coronary artery bypass surgery. Three patients were taking glyceryl trinitrate preparation, seven were taking lipid lowering treatment, two were on calcium antagonists, and five were on $\beta$ blockers. Three subjects had three vessel disease, two had two vessel disease, and four had one vessel disease. All had good left ventricular function with an ejection fraction greater than $50 \%$. One patient was diabetic and was on insulin, two had been classified as hypercholesterolaemic, and eight had been former smokers. Two patients entered the study during the early follicular phase, one in the late follicular phase, three in the early luteal phase, and two in the late luteal phase. No training effect between visits 1 and 4 was observed for time to ST segment depression, time to chest pain, or total exercise time. In the early follicular phase all subjects had low oestrogen and progesterone, while in the late luteal phase only one patient had a progesterone of less than $5 \mathrm{pmol} / 1$ and this patient had been luteal in the preceding week. All the other patients were appropriately in phase.
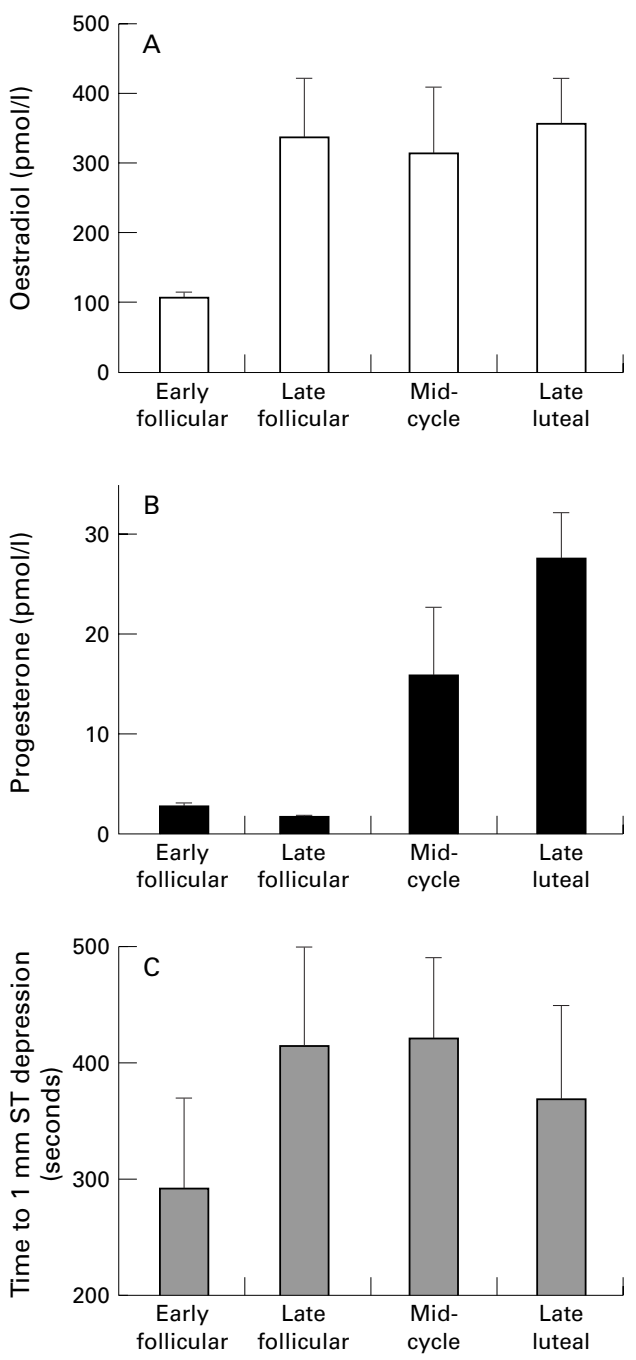

Figure 1 (A) Oestradiol (pmol/l); (B) progesterone (pmol/l); (C) time to $1 \mathrm{~mm}$ ST depression (with SEM) for each of the four phases of the menstrual cycle.

WEEK BY WEEK VARIATION

When the study period was divided into its constituent weekly visits, there was a significant effect of the different phases of the menstrual cycle on the onset of myocardial ischaemia, as assessed by time to $1 \mathrm{~mm} \mathrm{ST}$ segment depression (fig 1). During the period when oestrogen concentrations were lowest (early follicular), the time to ST depression was also at its lowest and it was significantly lower than in the late follicular period and in mid-cycle visits when oestradiol concentrations were higher (table 1). Total exercise time and time to onset of angina were highest in the late luteal phase when both oestradiol and progesterone concentrations were high, but although these differences were significant in pairwise comparisons, neither reached overall significance. Similar nonsignificant trends were observed in the maximum depth of ST segment depression. Examining the haemodynamic performance on the treadmill, the double product of systolic blood pressure and heart rate at the onset of myocardial ischaemia was also higher in the late follicular and mid-cycle visits, although again overall this did not reach significance. 
Table 1 Results of exercise treadmill testing for each phase of the menstrual cycle

\begin{tabular}{|c|c|c|c|c|c|c|c|}
\hline & Oestradiol (pmol/l) & $\begin{array}{l}\text { Progesterone } \\
\text { (pmolll) }\end{array}$ & $\begin{array}{l}\text { Double product at } 1 \mathrm{~mm} \mathrm{ST} \\
\text { depression }\end{array}$ & $1 \mathrm{~mm} S T$ depression & Angina (s) & $\begin{array}{l}\text { Exercise time } \\
(s)\end{array}$ & ST depth (mm) \\
\hline Early follicular & $104.6(11.5)$ & $2.5(0.6)$ & $18281(1880)$ & $290(79)$ & $379(82)$ & $453(75)$ & $1.9(0.3)$ \\
\hline Late follicular & $335.7(82.3)^{\star}$ & $1.5(0.2)$ & $21108(1364)$ & $412(87)^{\star}$ & $397(56)$ & $485(69)$ & $1.4(0.2)$ \\
\hline Mid-cycle & $313.0(89.6)^{\star}$ & $15.7(7.0)$ & $21565(1696)^{\star}$ & $418(71)^{\star}$ & $409(69)$ & $524(56)$ & $1.3(0.3)$ \\
\hline Late luteal & $352.5(66.2)^{\star}$ & $27.4(4.7)^{\star}$ & $18261(2045)$ & $366(82)$ & $487(81)^{\star \star}$ & $488(74)^{\star}$ & $1.5(0.2)^{\star}$ \\
\hline ANOVA & 0.08 & 0.07 & 0.18 & 0.02 & 0.17 & 0.27 & 0.20 \\
\hline
\end{tabular}

Values are mean $(\mathrm{SEM})$

${ }^{\star} \mathrm{p}<0.05,{ }^{\star \star} \mathrm{p}<0.01 v$ early follicular phase.

ANOVA, analysis of variance.

Table 2 Results for the 18 exercise tests performed in follicular and luteal phases (two tests per patient per phase)

\begin{tabular}{llll}
\hline Variable & Phase & Mean (SEM) & p Value \\
\hline Oestradiol (pmol/1) & $\begin{array}{l}\text { Follicular } \\
\text { Luteal }\end{array}$ & $206(46)$ & \\
& Follicular & $234(53)$ & 0.08 \\
Progesterone (pmol/1) & Luteal & $22(4)$ & \\
& Follicular & $19695(1178)$ & \\
Rate-pressure product (1 mm ST) & Luteal & $19913(1350)$ & 0.90 \\
$\quad$ (beats/min/mm Hg) & Follicular & $351(60)$ & \\
$\begin{array}{c}\text { Time to } 1 \mathrm{~mm} \text { ST depression } \\
\text { (min) }\end{array}$ & Luteal & $392(53)$ & 0.61 \\
Time to onset of chest pain (min) & Follicular & $388(48)$ & \\
& Luteal & $446(52)$ & 0.43 \\
Total exercise time (min) & Follicular & $469(49)$ & \\
& Luteal & $506(45)$ & 0.36 \\
\hline
\end{tabular}

LUTEAL VERSUS FOLLICULAR PHASE

Eighteen exercise tests in the nine patients were compared in both the luteal (high progesterone) and follicular (low progesterone) phases to explore the effect of progesterone on exercise performance. Time to onset of myocardial ischaemia, anginal chest pain, and total exercise time were not significantly different in either of these phases (table 2).

\section{Discussion}

It has been established that oestrogen and other female sex hormones have important effects on many different organ systems, including both the coronary and the peripheral arteries. ${ }^{14} 15$ The net result of oestrogen action is to cause vasodilatation, although this is antagonised to some extent by the progestin or progesterone. ${ }^{16}$ The mechanisms of this vasodilator effect have been examined in both ex vivo and in vivo models. Collins and colleagues showed that when oestradiol is injected into atheromatous human female coronary arteries, the atheromatous paradoxical constrictor effect in response to acetylcholine is reversed and the arteries dilate normally. ${ }^{14}$ This restoration of impaired endothelial function with augmentation of nitric oxide (NO) release from the endothelium is an important vasodilator reflex and will tend to increase coronary blood flow. Oestradiol at supraphysiological doses has also been noted to inhibit the influx of calcium ions into vascular smooth muscle cells, thus promoting vasodilatation, ${ }^{2}$ although the significance of this in vivo is not yet clear. Other mechanisms of vasodilatation include modification of the activity of angiotensin converting enzyme inhibition ${ }^{4}$ and antagonism of the constrictor effects of endothelin $1 .{ }^{17}$
Several published studies have suggested that postmenopausal oestrogen replacement may have antianginal effects, at least at supraphysiological doses, but this has not been universally accepted. Certainly the loss of female sex hormones at the menopause appears to be associated with a higher risk of cardiovascular disease, as shown by the increased cardiac risk among women undergoing early oophorectomy and the subsequent attenuation of this risk with hormone replacement therapy. ${ }^{18}{ }^{19}$ In a small case control study, premenopausal women with coronary disease had lower concentrations of oestradiol than healthy controls. ${ }^{20}$

In premenopausal women it is already established that blood pressure varies in a cyclical manner with fluctuations in the menstrual cycle, the highest pressure being observed in the luteal phase..$^{2122}$ There is a particularly steep rise as menstruation approaches, after which there is a return to baseline values. The release of NO shows clear evidence of periodicity with the menstrual cycle. Kharitonov and colleagues found that normal subjects at mid-cycle exhaled twice as much $\mathrm{NO}$ as in the perimenstrual period. ${ }^{9}$ Based on this evidence we hypothesised that cyclical variation in sex hormone concentrations might bring about alterations in the severity of anginal symptoms.

Exercise performance is a balance between symptomatic ischaemia and perception of symptoms. Sex hormones not only appear to modulate coronary tone, but can also influence pain perception and mood. In the early follicular phase when both oestradiol and progesterone concentrations were low, overall performance on the treadmill was at its worst. Myocardial ischaemia as judged by ST depression was significantly improved between this visit and the subsequent visits when oestradiol concentrations were higher. Over the four visits there was a significant effect of the menstrual phase. Time to onset of chest pain was also least in the perimenstrual period, although this was not significant. Total exercise time was unaffected. However, this dissociation between ischaemic markers and overall exercise performance is well reported. The variation in time to onset of ST depression may represent a loss of oestrogen mediated anti-ischaemic effect but could also represent alterations in autonomic tone that could in turn affect electrical repolarisation. It has been reported that false positive exercise tests occur more often in oestrogen positive women (that is, premenopausal or among those taking hormone replacement therapy). ${ }^{23}$ Similarly progesterone 
has been reported to affect the interpretation of exercise tests. ${ }^{24}$ In this study, when the population was divided into follicular (low progesterone) and luteal (high progesterone) phases no effect on treadmill performance was observed.

\section{CONCLUSIONS}

Our study is small in size and by its nature exploratory. The differences between the individual phases need to be interpreted with caution as there is some dissociation between clinical symptoms and treadmill derived ischaemia (this is particularly important because of the small sample size). Nevertheless the results suggest that myocardial ischaemia, as assessed on the treadmill, is worst in the perimenstrual period when both oestrogen and progesterone concentrations are at their lowest. This hypothesis of "menstrual angina" requires further evaluation and should be taken into account when interpreting symptoms and exercise tests in premenopausal women with angina.

We acknowledge the support of the Peel Medical Research Trust.

1 Collins P, Shay J, Jiang C, et al. Nitric oxide accounts for dose dependent oestrogen-mediated coronary relaxation
following acute oestrogen withdrawal. Circulation following acute

2 Zhang F, Ram JL, Standley PR, et al. 17- $\beta$ oestradiol attenuates voltage-dependent $\mathrm{Ca}^{2+}$ currents in $\mathrm{A} 7 \mathrm{r} 5$ vascular smooth muscle cell line. Am f Physiol 1994;266:C975-80.

3 Rosano G, Patrizi R, Leonardo F, et al. Effects of estrogen replacement therapy on heart rate variability and heart rate in healthy postmenopausal women. Am $\mathcal{F}$ Cardiol 1997;80 815-17.

4 Proudler A, Ahmed A, Crook D, et al. Hormone replacement therapy and serum angiotensin converting enzyme activity in postmenopausal women. Lancet 1996; 346:89-90.

5 Rosano GM, Sarrel PM, Poole-Wilson PA, et al. Beneficial effects of oestrogen on exercise-induced myocardial ischaemia in women with coronary artery disease. Lancet mia in women

6 Webb C, Rosano G, Collins P. Oestrogen improves exerciseinduced myocardial ischaemia in women. Lancet 1998:351: induced 1556 .
7 Holdright D, Sullivan A, Wright C, et al. Acute effects of oestrogen replacement therapy on treadmill performance in postmenopausal women with coronary artery disease. Eur Heart F 1995;16:1566-70.

8 Hashimoto M, Akishito M, Eto M, et al. Modulation of endothelium-dependent flow mediated dilatation of the brachial artery by gender and menstrual cycle [abstract]. Circulation 1995;92 (suppl I):I-430.

9 Kharitonov S, Logan-Sinclair R, Busset C, et al. Peak expiratory nitric oxide differences in men and women: relation to the menstrual cycle. Br Heart f 1994;72:243-5.

10 MacGregor EA, Chia H, Vohrah RC, et al. Migraine and menstruation: a pilot study. Cephalalgia 1990;10:305-10.

11 Skobeloff EM, Spivey WH, Silverman R, et al. The effect of the menstrual cycle on asthma presentations in the emergency department. Arch Intern Med 1996;156:183740 .

12 Rosano G, Leonardo F, Sarrel P, et al. Cyclical variation in paroxysmal supraventricular tachycardia in women. Lancet 1996;347:786-8.

13 Lomicek M, Proks V, Presl J. Coronary heart disease with a marked vasospastic component associated with the menstrual cycle and its treatment. Vnitrni Lekarstvi 1995;41: $710-12$

14 Collins P, Rosano GM, Sarrel PM, et al. 17- $\beta$-Estradiol attenuates acetylcholine-induced coronary arterial constriction in women but not men with coronary heart striction in women but not ment

15 Liebermann E, Gerhard M, Uehata A, et al. Estrogen improves endothelium-dependent flow mediated vasodilation in postmenopausal women. Ann Intern Med 1994;121: $936-41$

16 Rosano GM, Sarrel PM, Chierchia SL, et al. Medoxyprogesteron but not natural progesterone reverses the beneficial effect of estradiol 17-beta on exercise induced myocardial ischaemia [abstract]. Circulation 1996;91:I-18.

17 Jiang C, Sarrel P, Poole-Wilson P, et al. Acute effects of 17 beta estradiol on rabbit coronary artery contractile responses to endothelin 1. Am ₹ Physiol 1992;263(Pt 2): H271-5.

18 Colditz GA, Willett WC, Stampfer MJ, et al. Menopause and the risk of coronary heart disease in women. $N$ Engl f and the risk of coronary

19 Stampfer M, Colditz G. Estrogen replacement therapy and coronary heart disease: a quantitative assessment of the epidemiological evidence. Prev Med 1991;20:47-63.

20 Hanke H, Hanke S, Ickrath O, et al. Estradiol concentrations in premenopausal women with coronary heart disease. Coron Artery Dis 1997;8:511-15.

21 Greenburg G, Imeson J, Thompson S, et al. Blood pressure and the menstrual cycle. Br f Obstet Gynaecol 1985;92: 1010-14.

22 Manhem K, Jern S. Influence of daily-life activation on pulse rate and blood pressure changes during the menstrual cycle. F Hum Hypertens 1994;8:851-6.

23 Barrett-Connor E, Wilcosky T, Wallace R, et al. Resting and exercise electrocardiographic abnormalities associated with ex hormone use in women. Am f Epidemiol 1986;123:81-8.

24 Clark P, Glasser S, Lyman G, et al. Relation of results of exercise stress tests in young women to phases of the menexercise stress tests in young women to ph
strual cycle. Am 7 Cardiol 1988;61:197-9. 